\title{
EDITORIAL
}

\section{Warm up phenomenon and preconditioning in clinical practice}

\section{F Tomai}

Heart 2002;87:99-100

\begin{abstract}
More than half of all patients with coronary artery disease exhibit an improvement in performance following their first exercise test-the warm up phenomenon.
\end{abstract}

$\mathrm{T}$ he warm up phenomenon, first described more that 50 years ago in patients with effort angina, refers to the improved performance exhibited by more than half of all patients with coronary artery disease following their first exercise test.

There are several potential causes of the warm up phenomenon including: (1) an improvement in oxygen supply, which, in turn, may be caused by stenosis dilation, collateral recruitment or myocardial perfusion redistribution; (2) a reduction in oxygen consumption caused by an adaptive down regulation or stunning of regional myocardial contractile function in the ischaemic region; and (3) a form of myocardial adaptation to ischaemia akin to ischaemic preconditioning.

\section{MECHANISMS OF ADAPTATION TO EXERCISE INDUCED ISCHAEMIA}

It has been shown that in patients with a single lesion of the left anterior descending coronary artery, great cardiac vein flow is similar during the first and second exercise stress test, thus suggesting that the warm up phenomenon is not accompanied by an increase in total myocardial blood flow. ${ }^{2}$ These findings have been recently confirmed by Bogaty and colleagues, ${ }^{3}$ who showed that the attenuation of myocardial ischaemia observed with repeated exercise is not accounted for by an improvement in myocardial perfusion as determined by single photon emission computed tomography (SPECT) thallium-201 imaging. Unfortunately, both studies do not allow us to rule out the possibility that the warm up might be caused by myocardial blood flow redistribution from the epicardium to the endocardium.

Another potential mechanism of the warm up phenomenon is an adaptive down regulation of regional myocardial contractile function in the ischaemic region, which might diminish the severity of ischaemia through a reduction of oxygen consumption. In patients with stable angina Bogaty and colleagues ${ }^{4}$ found that ischaemia induced wall motion dysfunction during the second exercise was significantly attenuated compared with that observed during the first exercise, a finding consistent with the attenuation of electrocardiographic signs of ischaemia. More importantly, wall motion returned to initial baseline before the second test, thus suggesting that the attenuation of myocardial ischaemia observed with re-exercise could not be explained by down regulation of myocardial contractility induced by the initial ischaemic stimulus. Yet, significant wall motion abnormality persisted before the second exercise stress test when patients were challenged with more strenuous exercise, thus at least partially accounting for the attenuation of electrocardiographic ischaemic parameters and ischaemia induced contractile dysfunction observed during the second test. ${ }^{4}$ These findings were subsequently confirmed by Kelion and colleagues. ${ }^{5}$

"Another intriguing possibility is that the warm up phenomenon might be analogous to experimental ischaemic preconditioning"

Another intriguing possibility is that the warm up phenomenon might be analogous to experimental ischaemic preconditioning in which, in animal models, brief coronary artery occlusion preceding sustained occlusion, followed by timely reperfusion, can considerably reduce infarct size. ${ }^{6} \mathrm{~A}$ role for preconditioning is supported by the demonstration that the time course of the warm up phenomenon is consistent with that of ischaemic preconditioning (lasting no longer than between 60-90 minutes ). ${ }^{1}$ Indeed, the improvement of the ischaemic threshold observed during a second exercise test carried out within minutes of a first exercise test is lost during a third exercise carried out two hours after the second test.

\section{WARM UP PHENOMENON AND PRECONDITIONING}

Experimental ischaemic preconditioning refers to the ability of short periods of ischaemia to make the myocardium more resistant to a subsequent ischaemic insult. Although the complete signal transduction pathway of ischaemic preconditioning has not yet been elucidated, it is thought that binding of surface receptors by agonists, principally adenosine, released by the ischaemic myocardial cell results in activation of protein kinase $\mathrm{C}$ which in turn causes opening of mitochondrial ATP sensitive potassium $\left(\mathrm{K}_{\text {ATP }}\right)$ channels that may ultimately be responsible for the resistance to ischaemia. ${ }^{7}$ There is now evidence that preconditioning may also occur in patients in the setting of cardiac surgery, of coronary angioplasty, and of acute coronary syndromes. ${ }^{8}$ This notion is supported by the observation that in these human models, the adaptation to ischaemia is influenced by drugs acting on $\mathrm{K}_{\text {ATP }}$ channels and on purinergic receptors, similar to what is observed in 
accepted experimental models of ischaemic preconditioning.

Studies on $\mathrm{K}_{\text {ATP }}$ channel blockade of warm up by glibenclamide, at a dose previously shown to block adaptation to ischaemia during coronary angioplasty, ${ }^{9}$ have yielded conflicting results. In particular, in both diabetic ${ }^{10}$ and non-diabetic ${ }^{11}$ patients with chronic stable angina, glibenclamide prevented the increase of ischaemic threshold observed during the second of two sequential exercise tests, thus suggesting that ischaemic preconditioning plays a role in the warm up phenomenon and that in this setting it is, at least partially, mediated by activation of $\mathrm{K}_{\text {АтP }}$ channels. On the contrary, in two other studies in diabetic patients with chronic stable angina glibenclamide failed to prevent the warm up phenomenon. ${ }^{42}$ At present, therefore, the evidence for or against an involvement of $\mathrm{K}_{\mathrm{ATP}}$ channels in the warm up phenomenon remains uncertain.

Adenosine receptors have been identified as a key mediator in experimental ischaemic preconditioning and its clinical models of coronary angioplasty and coronary surgery. ${ }^{8}$ Yet both aminophylline, a non-selective antagonist of adenosine receptors, and bamiphylline, a selective antagonist of $A_{1}$ adenosine receptors, failed to prevent the warm up phenomenon. ${ }^{313}$

The elegant article published by Kelion and colleagues ${ }^{14}$ in this issue of Heart provides new insights into the pathophysiology of the warm up phenomenon. In fact, they investigated the effect of a selective $A_{1}$ adenosine receptor agonist, GR79236, on exercise induced ischaemia in patients with coronary artery disease undergoing sequential exercise tests. Their findings demonstrate that GR79236 failed to mimic the warm up effect, while warm up occurred even in its presence, therefore confirming that adenosine is unlikely to play an important role in this phenomenon. It is worth noting, however, that the cardioprotective effect of ischaemic preconditioning results from the combined action of several mediators, which together reach a threshold for cardioprotection. ${ }^{15}$ Thus, it is possible that, in the setting of the warm up phenomenon, the selective activation or blockade of one pathway only-that is, adenosine receptors-might be insufficient to mimic or prevent such a form of adaptation to ischaemia.

\section{IMPLICATIONS}

The mechanisms of the warm up phenomenon are probably multiple, and time and protocol dependent. The inconsistent results obtained in this setting with drugs which consistently mimic or prevent ischaemic preconditioning in humans in other clinical settings (coronary angioplasty, cardiac surgery, acute coronary syndromes) $)^{8}$ suggest that preconditioning might play a role in the warm up phenomenon, but it is mechanistically distinct from preconditioning caused by a sudden interruption of oxygen supply. Future work is warranted in order to understand better the mechanisms of the warm up phenomenon, including studies on transmural distribution of myocardial perfusion and on triggers of ischaemic preconditioning different from adenosine. At present, the current body of knowledge does not allow us to mimic the warm up phenomenon using drugs.

\section{REFERENCES}

1 Tomai F, Crea F, Danesi A, et al. Mechanisms of the warm-up phenomenon. Eur Heart J 1996;17:1022-27.

2 Okazaki Y, Kodama K, Sato H, et al. Attenuation of increased regional myocardial oxygen consumption during exercise as a major cause of warm-up phenomenon. J Am Coll Cardiol 1993;21:1597-604.

3 Bogaty P, Kingma JG, Guimon J, et al. Myocardial perfusion imaging findings and the role of adenosine in the warm-up angina phenomenon. J Am Coll Cardiol 2001;37:463-9.

4 Bogaty P, Kingma JG, Robitaille $M$, et al. Attenuation of myocardial ischemia with repeated exercise in subjects with chronic stable angina. Relation to myocardial contractility, intensity of exercise and the adenosine triphosphate-sensitive potassium channel. J Am Coll Cardiol 1998:32:1665-71.

5 Kelion AD, Webb TP, Gardner MA, et al. The warm-up effect protects against ischaemic left ventricular dysfunction in patients with angina. $J$ Am Coll Cardiol 200 1:37:705-10.

6 Marber MS, Joy MD, Yellon DM. Is warm-up in angina ischaemic preconditioning? Br Heart J 1994;72:213-5.

7 Cohen MV, Baines CP, Downey JM. Ischemic preconditioning: from adenosine receptor to K $\mathrm{ATP}_{\text {TP }}$ channel. Annu Rev Physiol 2000;62:79-109.

8 Tomai F, Crea F, Chiariello L, et al. Ischaemic preconditioning in humans. Models, mediators, and clinical relevance. Circulation 1999; 100:559-63.

9 Tomai F, Crea F, Gaspardone A, et al. Ischaemic preconditioning during coronary angioplasty is prevented by glibenclamide, a selective ATP-sensitive $\mathrm{K}^{+}$channel blocker. Circulation 1994:90:700-5.

10 Ovunc K. Effects of glibenclamide, a K-ATP channel blocker, on warm-up phenomenon in type II diabetic patients with chronic stable angina pectoris. Clin Cardiol 2000;23:535-9.

11 Tomai F, Danesi A, Ghini AS, et al. Blockade of ATP-sensitive K+ channels prevents the warm-up phenomenon. Eur Heart $J$ 1999;20:196-202.

12 Correa SD, Schaefer S. Blockade of $K_{\text {ATP }}$ channels with glibenclamide does not abolish preconditioning during demand ischemia. Am J Cardio 1997;79:75-8.

13 Tomai $F$, Crea F, Danesi $A$, et al. Effect of $A$ adenosine receptor blockade on the warm-up phenomenon. Cardiologia 1997;42:385-92.

14 Kelion AD, Webb TP, Gardner MA, et al. Does a selective adenosine A, receptor agonist protect against exercise induced ischaemia in patients with coronary artery disease? Heart 2002;87: 1 15-20.

15 Goto $M$, Liu Y, Yang XM, et al. Role of bradykinin in protection of ischemic preconditioning in rabbit hearts. Circ Res 1995;77:61 1-21.

\section{STAMPS IN CARDIOLOGY}

\section{British Heart Foundation}

Although no stamp has appeared which features the British Heart Foundation, the cover illustrated bears the slogan postmark for the Sixth World Congress of Cardiology held in London in September in 1970 and in addition advertises the British Heart Foundation. The stamp is the $4 \mathrm{~d}$ definitive in use at the time and the cover was franked in Liverpool, indicative of the national usage of this particular cancellation mark at the time.

M K Davies A Hollman

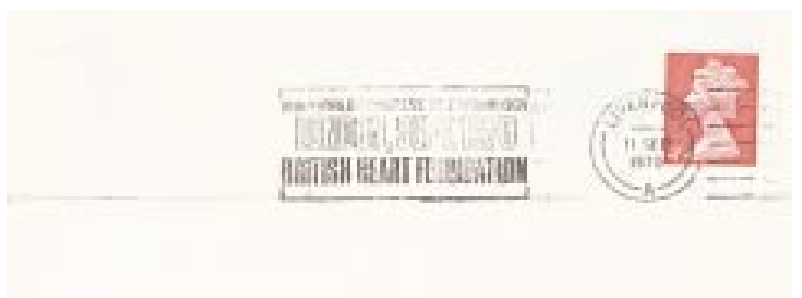

